\title{
Electrospun Biopolyesters as Drug Screening Platforms for Corneal Keratocytes
}

\author{
PEDRAM AZARI ${ }^{1}$, NG SOOK LUAN ${ }^{2}$, SENG NEON GAN $^{1}$, ROSIYAH YAHYA $^{1}, \mathrm{CHIOW} \mathrm{SAN} \mathrm{WONG}^{4}$, \\ KIEN HUI CHUA ${ }^{2}$, and BELINDA PINGGUAN-MURPHY ${ }^{3}$ \\ ${ }^{1}$ Department of Chemistry, Faculty of Science, University of Malaya, Kuala Lumpur, Malaysia \\ ${ }^{2}$ Department of Physiology, Faculty of Medicine, University Kebangsaan Malaysia, Jalan Raja Muda Abdul Aziz, Kuala Lumpur, Malaysia \\ ${ }^{3}$ Department of Biomedical Engineering, Faculty of Engineering, University of Malaya, Kuala Lumpur, Malaysia \\ ${ }^{4}$ Plasma Technology Research Center, Physics Department, Faculty of Science, University of Malaya, Kuala Lumpur, Malaysia
}

Received 3 October 2014, Accepted 14 March 2015

In vitro drug screening techniques provide rapid and easy to analyze data, while saving a lot of animals from being sacrificed. An important part of any in vitro drug screening platform is a biomaterial which promotes cell growth and proliferation. The potential of electrospun scaffolds made of polyhydroxybutyrate (PHB), poly (3-hydroxybutyric acid-co-3-hydroxyvaleric acid) (PHBV), and polycaprolactone (PCL) were studied to serve as drug screening platform for corneal keratocyte tissues. The results showed that the proliferation rate was slightly higher for PCL and PHBV on day 7. Gene expression results showed that PCL was the best in maintaining keratocyte genes.

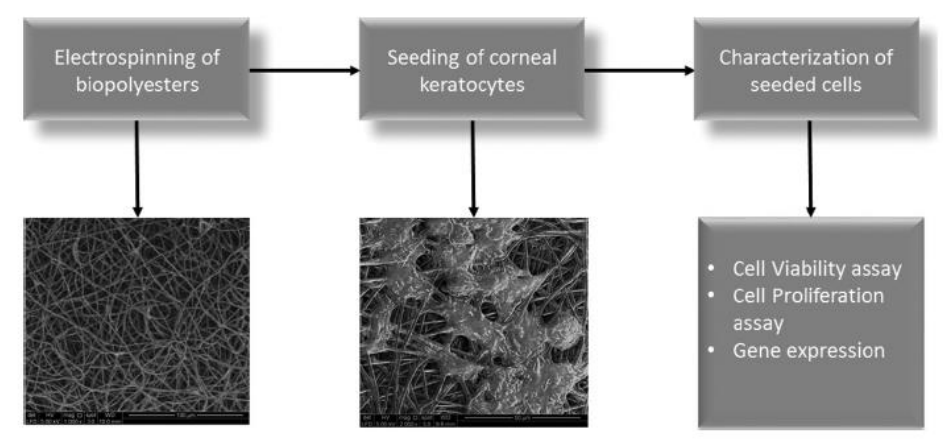

Keywords: Biopolyesters, corneal keratocytes, electrospinning, polycaprolactone, polyhydroxybutyrate, polyhydroxybutyrate-cohydroxyvalerate

\section{Introduction}

Infection, trauma, viral, and atopic disease as well as genetic deficiencies are just some of the causes for corneal function disorders [1,2] that lead to corneal blindness of more than 45 million individuals all around the world annually [3]. Although mild injuries can be cured by the natural immune

Address correspondence to: Belinda Pingguan-Murphy, Department of Biomedical Engineering, Faculty of Engineering, University of Malaya, 50603 Kuala Lumpur, Malaysia. E-mail: bpingguan@gmail.com

Color versions of one or more of the figures in the article can be found online at www.tandfonline.com/gpom. system response or via clinically available techniques, severe cases still await the development of novel drugs [4]. In order to assess the toxicity of any novel treatment, animal experimentation is needed. Besides being costly, animal experimentation requires a large number of animals to be sacrificed [5]. As an alternative to animal tests, in vitro drug screening could be considered, a method that has the benefits of ease of application, simplicity of testing, and straightforward data interpretation as well as not involving animals. It provides rapid and cost-effective screening with good sensitivity [6]. Although accurate extrapolation of the in vitro results to in vivo conditions remains challenging, it can still be very useful for preliminary stages of studies and saves a lot of animals. 
In order to undertake such in vitro drug testing for application to the cornea it is necessary to have a reliable scaffold material capable of promoting and maintaining the growth of keratocytes, the cells which make up about $90 \%$ of the volume of corneal stroma, being bonded to an extracellular matrix of highly regular collageneous lamella.

In this research, we have developed electrospun scaffolds for this purpose from three commercially available biopolyesters and studied their potential to promote corneal keratocyte growth and characteristics.

Notably, keratocytes usually stay in the quiescent state and maintain noncrystalline structures to make the corneal transparent and have optimal refraction. However, they are able to undergo transition into repair phenotypes, namely activated fibroblasts or myofibroblasts in respond to specific environmental signals, example injury and wound healing [7]. It is important for keratocyte to differentiate because it helps in retaining corneal transparency and determining the corneal response to injury [8]. This clearly established that keratocytes are sensitive to the changes in environmental condition, and therefore understanding the behaviors of keratocytes in different testing conditions will greatly increase our knowledge in corneal wound healing and regeneration.

Concerning the use of biopolyesters, it is notable that these have been used previously as cell carriers for biomedical applications, suggesting a possible application here as well [9-12]. Advantageously, these polymers have a low cost of synthesis, degrade easily through hydrolysis of ester bonds, and produce nontoxic degradation products [13,14]. Production of scaffold is via electrospinning technology, which allows fabrication of a controllable fibrous scaffold similar to the natural extracellular matrix (ECM) $[15,16]$, in a simple, versatile, and cost-effective manner. In the electrospinning process micro and nano fibers are produced by stretching a polymeric solution by high electric field $[17,18]$. There are a number of processing parameters that can affect the morphology of the fibers, which include the chemical nature of the polymer, solvent, applied voltage, spinneret to collector distance, feeding rate, and capillary diameter. Based on the applied parameters the morphology can be varied to produce uniform fibers, beaded fibers, and fibers with spindles on string [19]. In this comparative study we have subjected three different biopolyesters to the same electrospinning parameters and obtained different morphologies, presumably due to the different chemical natures of the polymers. The potential of these materials in terms of corneal keratocyte proliferation and differentiation were then investigated.

\section{Experimental}

\subsection{Chemicals}

Poly[(R)-3-hydroxybutyric acid] (PHB), poly(3-hydroxybutyric acid-co-3-hydroxyvaleric acid) (PHBV), and polycaprolactone $(\mathrm{Mn}=80000 \mathrm{~g} / \mathrm{mol})$ (PCL) were purchased from Aldrich. F12: DMEM (FD), antibioticantimycotic, trypsin-EDTA glutamax, and fetal bovine serum were purchased from Gibco Invitrogen, USA. 3(4,5-dimethylthiazolyl-2)-2,5-diphenyltetrazolium bromide solution was purchased from Sigma Aldrich USA. Vitamin C, dimethyl sulfoxide, paraformaldehyde, chloroform, dimethylformamide (DMF), and phosphate buffer saline were purchased from Merck Millipore, Germany. Alamar blue solution was purchased from Life Technologies, USA. TRI Reagent and polyacryl carrier were purchased from Molecular Research Center, USA. DNAse- and RNAsefree distilled water and SuperScript III First-Strand Synthesis SuperMix kit were purchased from Invitrogen, USA.

\subsection{Electrospinning}

For ease of comparison the same parameters were applied to PHB, PHBV, and PCL. A $10 \%(\mathrm{wt} / \mathrm{v})$ solution of polymer was prepared in a co-solvent mixture comprising of chloroform and DMF with a ratio of 9:1. The solution was stirred at $60^{\circ} \mathrm{C}$ in a round bottom flask fitted with a condenser for $5 \mathrm{~h}$ to reach homogeneity. The solution was loaded into a $20 \mathrm{~mL}$ syringe (Terumo) placed $18 \mathrm{~cm}$ away from the collector horizontally. The feeding rate of the syringe pump was set at $3 \mathrm{~mL} / \mathrm{h}$. A semi rectified DC voltage of $12 \mathrm{kV}$ was generated from a custom-made high voltage power supply to conduct electrospinning.

\subsection{Field Emission Scanning Electron Microscopy}

The morphology of the electrospun fibers and cells were studied by using field emission scanning electron microscopy (FEI: QUANTA FEG 250). Average diameter of the fibers for each sample was measured using ImageJ software. For each sample 25 measurements was carried out at different random spots. Fiber intersections and beads on fibers were excluded from measurements. For samples seeded with cells, cell fixation was carried out prior to observation. Cells were fixed with a $4 \%$ paraformaldehyde after removal of the culture medium followed by washing in PBS. Field emission scanning electron microscopy was conducted without applying any coating on the samples. The samples were studied on days $1,3,5$, and 7 .

\subsection{Cell Culture}

Six New Zealand white rabbits' corneal tissues were obtained from the local animal slaughterhouse. The corneal tissues were processed using the techniques reported by Ghafar et al. [20]. The use of corneal cells for research purpose was approved by the Universiti Kebangsaan Malaysia animal ethic committee with approval number of: FF092-2012. Keratocytes were harvested from the corneal stroma with $0.3 \%$ collagenase type I digestion as mentioned in a recent paper [4]. Cell cultures were maintained in F12: DMEM (FD) media supplemented with $10 \%$ fetal bovine serum (Gibco Invitrogen, USA), 1\% antibiotic-antimycotic, $1 \%$ glutamax, and $1 \%$ vitamin $\mathrm{C}$ under standard incubation at $37^{\circ} \mathrm{C}, 5 \% \mathrm{CO}_{2}$, and $95 \%$ humidity. Primary culture (P0) was passaged at a split ratio of 1:4 when cells reached 
approximately $90 \%$ confluence using $0.125 \%$ trypsin-EDTA Passage 1 culture was used in the study.

\subsection{Leachate Preparation}

Leachate was prepared at a surface-area-to-extractant volume ratio based on ISO 10993-12. The ratio was $120 \mathrm{~cm}^{2}$ per $20 \mathrm{~mL}$ extraction vehicle when the material thickness is less than or equal to $0.5 \mathrm{~mm}$. The scaffolds (PCL, PHB, and $\mathrm{PHBV}$ ) with size $2.27 \mathrm{~cm}^{2}$ were prepared and disinfected with $70 \%$ ethanol. Twenty scaffolds were rinsed with PBS $1 \mathrm{X}$ thrice and incubated in FD media at $37^{\circ} \mathrm{C}$ and $5 \%$ $\mathrm{CO}_{2}$ for 3 days.

\subsection{Cell Viability and Cytotoxicity Test}

Cytotoxicity of scaffold leachate was assessed via 3-(4,5 dimethylthiazolyl-2)-2,5-diphenyltetrazolium bromide (MTT) assay. Passage 1 keratocytes with the seeding density of 50,000 cells $/ \mathrm{mL}$ were seeded onto 96-well culture plate overnight. The keratocytes were treated with 10 leachate concentrations using a dilution factor of 10 in FD media for $72 \mathrm{~h}$. MTT solution was added and incubated for $4 \mathrm{~h}$ at $37^{\circ} \mathrm{C}$ and $5 \% \mathrm{CO}^{2}$. Dimethylsulfoxide (DMSO) was added to dissolve the purple formazan before reading its absorbance at wavelength $570 \mathrm{~nm}$.

\subsection{Cell Proliferation Assay}

The Alamar Blue assay was used to measure the cell proliferation continuously since the cells remained viable after performing the cytotoxicity test. Passage 1 keratocytes with a seeding density of $3.5 \times 10^{4}$ cells were seeded onto the scaffolds (PCL, PHB and PHBV) respectively for 4 hours. Scaffolds were rinsed with PBS X1 in order to remove the unattached cells, then the scaffolds were transferred into new culture wells with $2 \mathrm{~mL}$ FD $+10 \%$ FBS medium. On day 1 Alamar Blue assay, the culture media were removed and substituted with $550 \mu \mathrm{L}$ medium containing $10 \%$ Alamar blue. After $4 \mathrm{~h}$ incubation at $37^{\circ} \mathrm{C}$ and $5 \% \mathrm{CO}_{2}, 200$ $\mu \mathrm{L}$ of medium was aspirated and read using ELISA microplate reader with wavelength $570 \mathrm{~nm}$. The Alamar Blue test was repeated for cultures for days 3,5 , and 7 . On day 7 , the keratocytes cultured on the scaffolds were harvested using Trypsin-EDTA $0.125 \%$ after the Alamar Blue assay. Keratocytes were centrifuged and the pellet was preserved using TRI Reagent (Molecular Research Center, Cincinnati, USA) for total RNA extraction. A standard curve was plotted based on the Alamar blue absorbance reading in serial dilutions of cell number to obtain an equation, and to calculate the number of viable cells.

\subsection{Total RNA Extraction and Two-Step Reverse Transcriptase Polymerase Chain Reaction (RT-PCR)}

Total RNA extraction was carried out according to the manufacturer's protocol. Polyacryl carrier (Molecular Research Center, USA) was used for total RNA precipitation. The total RNA pellet was washed with $75 \%$ ethanol and air dried before dissolving in DNAse- and RNAse- free distilled water
(Invitrogen, Carlsbad, CA, USA). The purity and concentration of extracted total RNA was determined by Nanodrop ND-100 spectrophotometer (Wilmington, DE, USA) and stored at $-80^{\circ} \mathrm{C}$ for further use. The synthesis of complementary DNA was performed using SuperScript III First-Strand Synthesis SuperMix kit (Invitrogen) according to the manufacturer's instruction. The reverse transcription was carried out at $50^{\circ} \mathrm{C}$ for $30 \mathrm{~min}$. The forward and reverse primers used in the quantitative RT-PCR were designed from the sequences obtained in NIH Genbank database using Primer-3 software as published in [4]. Glycerylaldehyde-3-phospate dehydrogenase (GAPDH) was used as housekeeping gene for the data normalisation. Two-step RT-PCR was carried out using iQSYBR Supermix in Bio-Rad iCycler (Bio-Rad, USA). The protocol conditions were initiated with the activation of Taq DNA polymerase at $94^{\circ} \mathrm{C}$ for 3 min, followed by 45 cycles of PCR amplification at $94^{\circ} \mathrm{C}$ for $10 \mathrm{~s}$ and $60^{\circ} \mathrm{C}$ for $30 \mathrm{~s}$ and then melting curve analysis. The specificity and size of PCR products were confirmed with $2 \%$ agarose gel electrophoresis.

\subsection{Statistical Analysis}

Quantitative data were obtained for six samples and tested for statistical significance using Statistical package for Social Sciences (SPSS) version 20. The results were presented in groups as mean \pm standard error of mean (SEM). One-way analysis of variance (ANOVA) was performed in normally distributed data, while Kruskal-Wallis test was used as nonparametric test. Post hoc tests were Tukey HSD and Mann-Whitney. $\mathrm{P}<0.05$ was considered to be statistically significant.

\section{Results and Discussion}

\subsection{FESEM}

Figure 1 shows a microscopic view of blank electrospun scaffolds. As the image suggests, PCL leads to the formation of fibers with a smaller diameter than those of PHB and PHBV under the same conditions. PCL, due to its lower molecular weight, resulted in less viscose solution with less chain entanglement (Figure 1a). Therefore, during electrospinning it could be stretched to a much lower diameter. However, a lower viscosity could result in less stable jet, with formation of more random fibers, and formation of beads within the electrospun fibers [21]. In agreement with this, fiber diameter distribution patterns of the fibers (Figure 1) confirm PCL exhibits the lowest diameter, at $300 \mathrm{~nm}$, followed by PHB at $1.12 \mu \mathrm{m}$ and PHBV at $1.35 \mu \mathrm{m}$. PHB, comparatively, is more rigid and stiff because of its high crystallinity, formed more straight fibers [22] (Figure 1b). As a result of the incorporation of hydroxyvalerate into PHBV, the polymer is also more elastic. PHBV fibers show good uniformity as well as porosity, and have a looped shape rather than just being straight.

Figure 2 shows cell morphology and cell to scaffold interaction at four different days: 1, 3, 5, and 7. The SEM images of cells show cells growing on the electrospun scaffolds. 

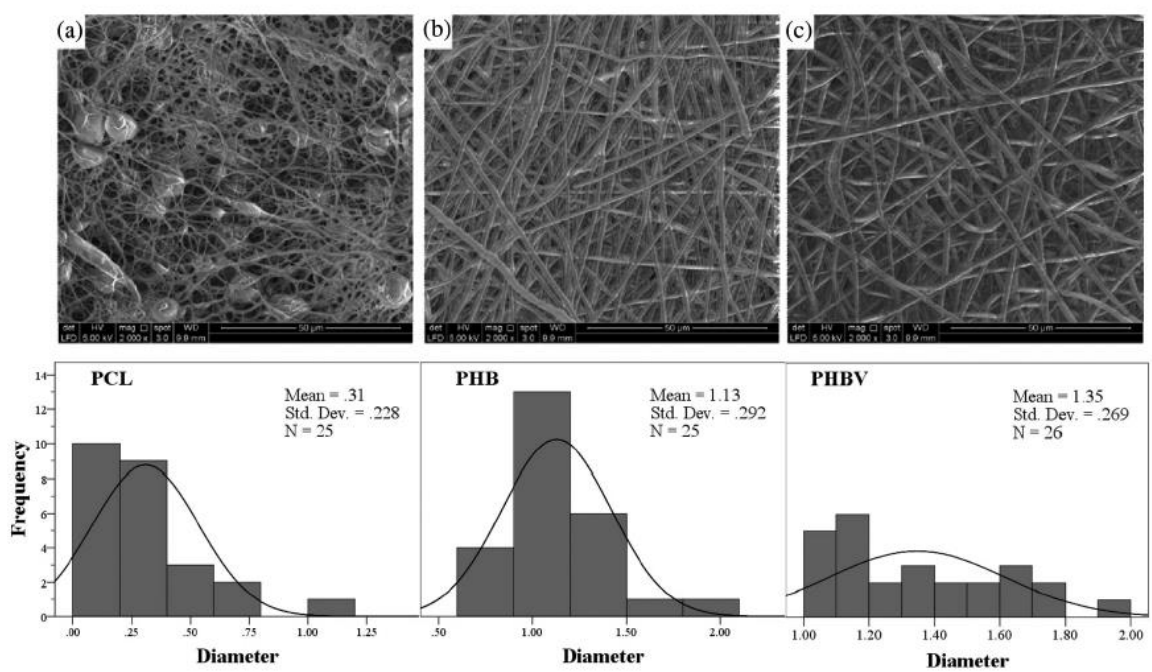

Fig. 1. FESEM microscopy of the blank scaffolds (a) PCL, (b) PHB, (c) PHBV, and their relevant fiber diameter distribution pattern.

These cells can spread and penetrate into the fibers. The filopedia extend along the fibers on all the scaffolds, as guided by the orientation of the fibers. In PHB scaffolds, the micrographs showed the cells were larger, flatter and stretched.
This latter observation may indicate higher expression of $\alpha$ -SMA2 on PHB. During corneal wound healing, keratocytes are activated and transformed into fibroblasts and eventually become alpha-smooth muscle actin ( $\alpha$-SMA)-expressed
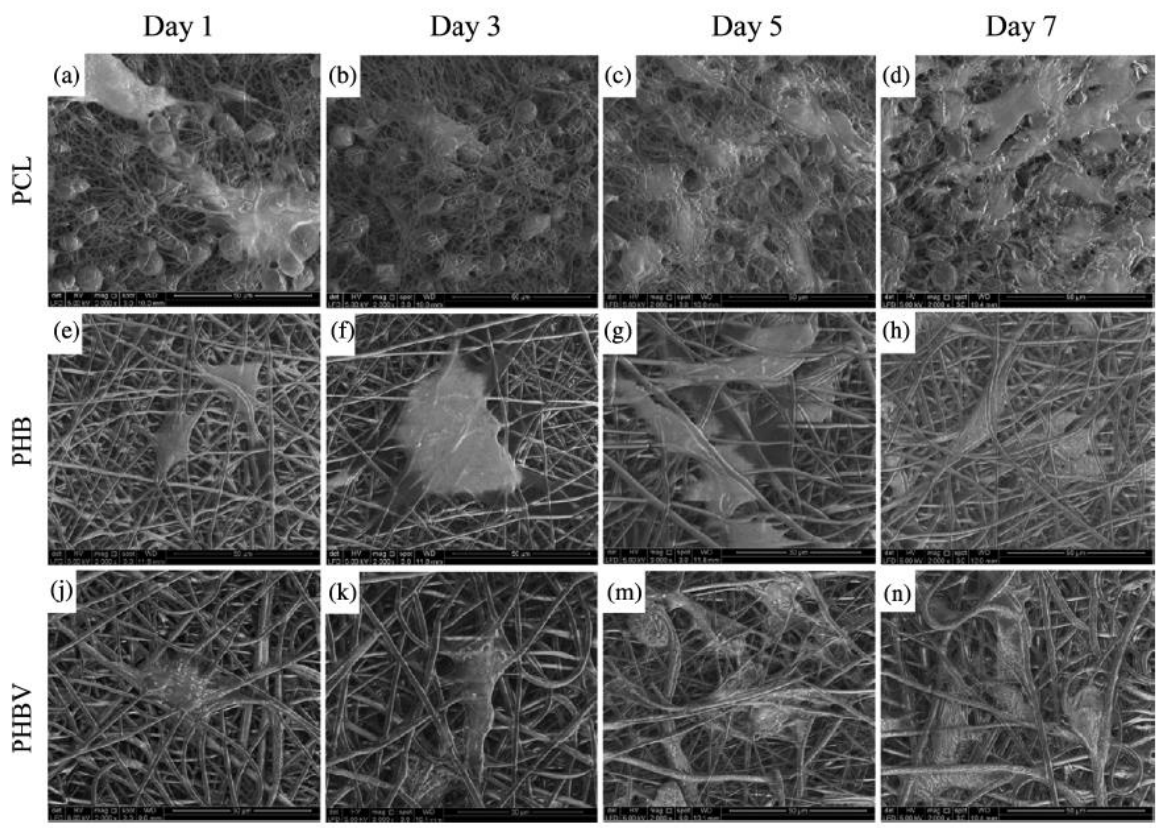

Fig. 2. FESEM images showing the morphology of keratocytes cultured on PCL (a-d) days 1-7, PHB (e-h) days 1-7, and PHBV $(\mathrm{j}-\mathrm{n})$ days $1-7$ with magnifications of $2000 \times$.

Link to full text journal article :

http://www.tandfonline.com/doi/pdf/10.1080/00914037.2015.1030648

http://www.ingentaconnect.com/content/tandf/gpom/2015/00000064/00000015/art00003 